\title{
Have Astronauts Visited Neptune? Student Ideas About How Scientists Study The Solar System
}

\author{
Christopher Palma, Pennsylvania State University, USA \\ Julia Plummer, Pennsylvania State University, USA \\ KeriAnn Rubin, St. Ann School, USA \\ Alice Flarend, Bellwood-Antis School District, USA \\ Yann Shiou Ong, Pennsylvania State University, USA \\ Scott McDonald, Pennsylvania State University, USA \\ Chrysta Ghent, Pennsylvania State University, USA \\ Timothy Gleason, Pennsylvania State University, USA \\ Tanya Furman, Pennsylvania State University, USA
}

\begin{abstract}
The nature of students' ideas about the scientific practices used by astronomers when studying objects in our Solar System is of widespread interest to discipline-based astronomy education researchers. A sample of middle-school, high-school, and college students $(N=42)$ in the U.S. were interviewed about how astronomers were able to learn about properties of the Solar System as a follow-up question after specific questions about the nature of the Solar System and its objects. These students often held naive ideas about the practices of astronomy, and 19\% of them proposed that humans or robots have returned samples of the planets to Earth for analysis. While the college students provided more sophisticated responses to the questions than the younger students, we found that even they held naive ideas about human sample return and infrequently appealed to studying objects at a distance using telescopes. We propose that students are not receiving specific instruction that allows them to investigate the tools and practices of astronomy, which leads them to rely on their prior knowledge about science practices in other disciplines (e.g., geoscience) when queried about how scientists study the Solar System. This result implies that instruction around the limits of human and robotic spaceflight is needed to allow students to have a more scientific understanding of the practices of astronomy in studying the Solar System.
\end{abstract}

Keywords: Astronomy Education; Solar System

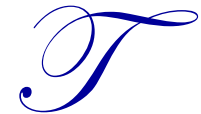

he National Research Council's Framework for K-12 Science Education Framework (NRC, 2012) recommends science teaching be organized around big ideas in a way that also integrates student engagement in scientific practices. Studies like those by Kesidou and Roseman (2002) consistently suggest that most science teaching does not follow these recommendations; students are instead asked to memorize facts and study phenomena without enough support to see how these may be related. The Framework offers a potential solution by presenting a vision for science education organized around three dimensions: 1) Scientific and Engineering Practices, 2) Crosscutting Concepts, and 3) Disciplinary Core Ideas. More importantly, it emphasizes that these three dimensions must be integrated as this best reflects the nature of the scientific discipline. That is, the emphasis is on students participating in investigations that are designed to both reveal the content knowledge of a discipline while simultaneously engaging students in the practices of the discipline under study. The practices of scientists in a particular discipline area (e.g., astronomy) can vary dramatically from those in other areas (e.g., physics). Therefore, students without any foundation in the practices of a particular discipline will be limited in the extent to which they can rely on their experience with the practices of a more familiar discipline to help them reason out the answer to a problem in a less familiar discipline. Thus, the Framework stresses how engagement in scientific practices is part of the progression of learning in every discipline; using scientific practices to perform investigations throughout the K- 
12 curriculum will allow students to delve more deeply into science at higher grade levels than they otherwise would without direct experience with those practices.

Songer and Gotwals (2012) investigated methods of assessing student learning in a way that fuses core content ideas and science practices in biodiversity. They emphasize that standards documents are often written with the core ideas and practices listed as separate standards. This approach leads to instruction and assessment of these to also be separated, even though the emphasis in the standards documents is on the fusion of these in modern science instruction. Songer and Gotwals note that, other than their work in biodiversity, there have been few studies of the fusion of core content ideas and science practices in a particular content domain. And despite many domain-general characteristics of science practices, students' ability to use science practices are often domain-specific, through their dependence on domain-specific prior knowledge (Eberbach \& Crowley, 2009; NRC, 2007). Therefore, we set out to begin to fill this gap by assessing students' ideas at the intersection between content and practice in the domain of astronomy.

\section{Practices of Science in Astronomy}

Astronomy is primarily an observational science (in contrast to an experimental science) that often requires the use of telescopes to observe objects at immense distances to gather evidence to support scientific claims. Prior research has shown that the linear, step-by-step presentation of the scientific method presented in most textbooks differs dramatically from how scientists describe their methods (e.g., Reiff, Harwood, \& Phillips 2002); further, Lowman (2002) makes the argument that astronomy, as a primarily observational science, strongly deviates from the norms expected by many K-12 classroom teachers more familiar with the experimental methods used in other disciplines. For example, astronomers are unable to set up experiments with controls and to vary a single variable, but must instead observe the experiments that the Universe has set up through the natural evolution of astronomical objects and systems. Thus, astronomical investigations often look nothing like a typical chemistry experiment, for example, which can lead to challenges in designing curricula that accurately reflect how astronomy is practiced.

Professional astronomers make use of a variety of instruments to make their observations, including enormous groundbased telescopes with apertures of 10 meters or more, sophisticated space-based telescopes like the Hubble Space Telescope, and special purpose ground-based or space-based telescopes that make use of an invisible part of the electromagnetic spectrum such as radio waves, infrared, or gamma-rays. In our teaching, we have had students describe their picture of an astronomer as a person peering through a small telescope with their eye; however, all modern research instruments use advanced digital detectors (which are essentially just sophisticated cameras) to record data for later quantitative analysis by computers, rather than by eye.

Because objects within our own Solar System are within the reach of our space travel technology, we are able to study these objects in more detail than we can just with telescopes alone. Astronomers who study the Solar System, known as planetary scientists, make use of robotic orbiters and landers that are sent directly to other worlds for more detailed measurements of these objects to supplement what we can learn about them from Earth-based or space-based (but still Earth-orbiting) telescopes. Planetary scientists are also able to investigate questions about the Solar System directly by analyzing meteorites, including lunar and Martian meteorites, which can be studied in great detail in Earth-based laboratories. Astronomers who study the Sun are referred to as heliophysicists, and their practices overlap significantly with those of planetary scientists. On the other hand, astronomers who study more distant objects, such as stars and galaxies, have no choice but to only use telescopes to study their targets. This difference in scientific methodology, based on the difference in distance between the objects they study, is part of the reason planetary scientists are unique in the field of astronomy. An example of this difference is illustrated by Figure 1, which contrasts the best image of Pluto as seen from the Hubble Space Telescope with an image of Pluto taken from the New Horizons flyby mission; there are many more types of scientific questions about the nature of Pluto that can be undertaken with the New Horizons data than the Hubble data, although both are quite valuable scientifically. 
Figure 1. Image of Pluto and several of its moons as observed by the Hubble Space Telescope (left panel; credit: NASA, ESA, H. Weaver (JHU/APL), A. Stern (SwRI), and the HST Pluto Companion Search Team) compared to a much higher resolution image of part of Pluto's surface as observed by the New Horizons flyby mission (right panel; credit: NASA/Johns Hopkins University Applied Physics Laboratory/Southwest Research Institute).

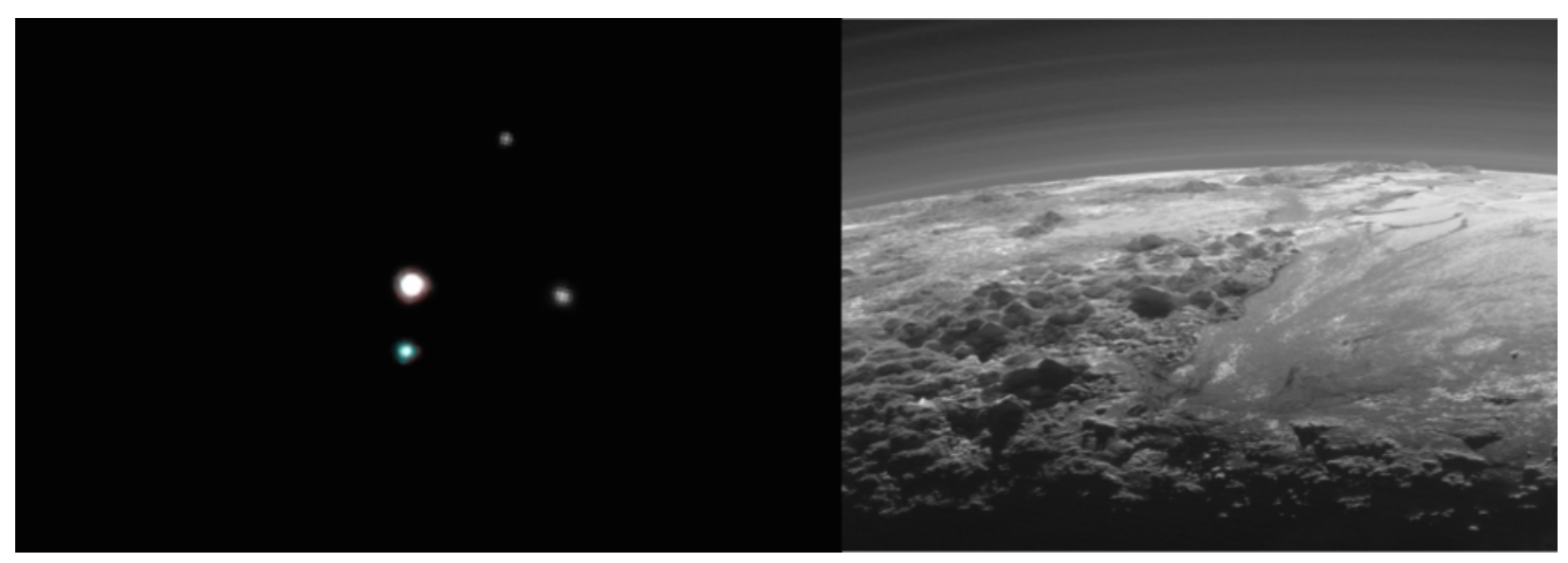

These complexities in what it means to carry out astronomical research suggest two areas of potential challenge for student understanding. First, research on students' ideas about astronomical scales has direct implications for our study of their understanding of astronomical practices (e.g., Miller \& Brewer 2010). Students who dramatically underestimate the distances to the stars will not see the need for different methods of investigating stars if they believe they are just as easy to reach as the planets in our Solar System. Second, the practice of using both ground-based and space-based telescopes to obtain images is complicated; yet, many students have experience simply retrieving these images from the internet without needing to understand how a telescope and camera (or other astronomical detector) were used to obtain them in the first place.

The research work of a professional astronomer who uses the tools and techniques discussed above maps well onto the "Practices for K-12 Science Classrooms" in the Framework. In the research we describe here, we are focusing in particular on just two of these practices: (1) how astronomers plan and carry out their investigations and (2) how they analyze and interpret their data. Astronomers have to carefully plan investigations to take the most efficient advantage of limited telescope resources. Telescopes and computer models create data sets that are analyzed for months or years to provide evidence that addresses the research questions being investigated. The Next Generation Science Standards (NGSS; NGSS Lead States, 2013) proposes that students as young as the middle-school students in our sample are expected to learn these practices. The NGSS includes Earth and Space Science standard MS-ESS1-3, which is: "Analyze and interpret data to determine scale properties of objects in the Solar System". They further clarify this with the statement, "Emphasis is on the analysis of data from Earth-based instruments, space-based telescopes, and spacecraft to determine similarities and differences among Solar System objects."

\section{Student Ideas and Opportunities for Learning}

There is some evidence suggesting students see the practices of astronomy differently than the practices of other sciences (e.g., Brickhouse et al., 2002) and, in particular, that they find the claims of astronomy less believable than biology, for example. In the Brickhouse and colleagues' (2002) study, U.S. college students taking an astronomy course for non-majors expressed skepticism about astronomical claims because of the methods used to collect astronomical data on objects at a distance and the inherent uncertainties involved in studying objects that can never be physically manipulated in a laboratory. While students' experience using scientific equipment to make measurements varies widely, in our experience students have far less exposure to investigations that expose them to the practices of astronomy than they do performing biology, chemistry, or physics experiments. Furthermore, in most U.S. K-12 curricula, these three disciplines receive far more instructional time than astronomy. 
Although there are opportunities for middle- and high-school students to engage directly in astronomical research using data from modern observatories, e.g., the NITARP program (Deeb et al., 2015) and Advanced Astronomy Camp (Fields 2009), this is incredibly rare in U.S. classrooms. Instead, students either get no direct access to a telescope during their study of astronomy, or they may have the opportunity to spend a minute or so looking through an eyepiece of a small telescope at a bright object like the Moon or Jupiter. Thus, few students are experiencing the type of astronomy instruction recommended by the Framework, where they would be directly engaging in a critical aspect of the practices of astronomy while studying this content area. Although rare, there have been a number of small, local projects, as well as projects with a more national scope, designed to give students more exposure to telescopes. These often make use of robotic telescopes in distant locations (viz., e.g., Sadler et al., 2001, Gehret et al., 2004, Camacho et al., 2009), which allows students to have the opportunity to collect data from a location where it is nighttime while they are in school during normal daytime hours. There have also been efforts, such as Project CLEA (Marschall et al., 2000), to create very realistic simulators to allow students to simulate the experience of obtaining their own astronomical observations from a research grade observatory. However, these instructional opportunities have not been examined in ways that would reveal students' understanding of the practices of science used by astronomers.

Much of the literature that exists on K-12 student use of telescopes provides information on how these systems are created or adapted for ease of student use (e.g., Hoban et al., 2006), or how exposure to real astronomical telescopes may improve attitudes towards science (e.g., Ferlet \& Pennypacker 2006). Some authors discuss how telescope use appears to influence student learning (Gehret et al., 2004). Gould and colleagues (2006) did a quantitative analysis of students' performance on the Project STAR Astronomical Concept Inventory before and after their use of the MicroObservatory telescopes. They saw improvement in student understanding of concepts related to light and size and scale, which were emphasized in the investigations students performed as part of the MicroObservatory curriculum. Yet, their results did not illuminate how this experience improved students' understanding of astronomical practices. Overall, there has been little research on how to support students in learning these practices of astronomy, whether it is through direct, remote, or simulated use of telescopes as part of an astronomy curriculum (Gershun, Slater, \& Berryhill, 2014; Slater et al., 2014). Further, we did not find any prior studies that provide an in depth description of student understanding of the practices of astronomy, which is an important first step before looking at how engagement in these practices supports student learning of the fusion of astronomy practices with astronomy content.

Many K-12 astronomy curricula and state standards documents emphasize Solar System astronomy (Palen \& Proctor, 2006). Given the gap we identified in the literature around astronomical practices and the widespread focus on Solar System phenomena in K-12 classes, our study was guided by the following research question: What are students' ideas about the scientific practices used by astronomers in their study of the properties of objects that make up the Solar System?

\section{METHODS}

This work is part of a larger empirical study of student learning of Solar System Astronomy. In a previous part of this study, we collected student interviews to create a hypothetical learning progression in Solar System astronomy (Plummer et al., 2015). The current study makes use of student responses that were not analyzed in that previous publication. Students in middle school (6th grade; $n=18)$, high school $(9$ th -12 th grade; $n=18)$, and college $(n=6)$ from urban, suburban, and rural locations in Pennsylvania were interviewed $(\mathrm{N}=42)^{1}$. The middle and high school students were selected randomly, and no effort was made to pick students who had recent or ongoing astronomy instruction. On the other hand, the college students were selected from students who had completed an introductory astronomy course to include a subsample of students who had specific prior experience with astronomy instruction, and could therefore provide us with more sophisticated answers to compare to those of the younger students in the study.

\footnotetext{
${ }^{1}$ In the Plummer et al. (2015) study, the sample size was reported as $\mathrm{N}=44$. However, two of the students in that study were asked only astronomy content questions and were not asked the questions about science practices necessary to address this research question, so their responses were not analyzed in this study.
}

Copyright by author(s); $\underline{\mathrm{CC}-\mathrm{BY}}$ 
The open-ended interview protocol engaged students in describing the current structure and motion of objects in the Solar System, formation of the Solar System, and gravity (analyzed for Plummer et al., 2015). All of the questions analyzed in this study are summarized below in Table 1. The questions listed in Table 1 are the top-level questions that were posed to the student. After the student provided an answer, if they had not already explicitly addressed this in their response, the interviewer followed up with the question "What did scientists see or measure to figure that out?" or a close variation of that wording. Some of the questions in the table presuppose that the student provided a normative answer, such as question 2 about the motion of the moon and question 5 about the flatness of the Solar System. However, if the student did not provide a normative answer, we would provide them some guidance about the normative answers by saying something similar to "Experts have said that the Solar System is flat, have you heard that before?" We would then follow up with the question about what scientists see or measure.

For this analysis, we coded only the sections pertaining to students' responses about how scientists investigate the Solar System phenomena. The whole interview protocol is available upon request.

Table 1. Interview protocol questions

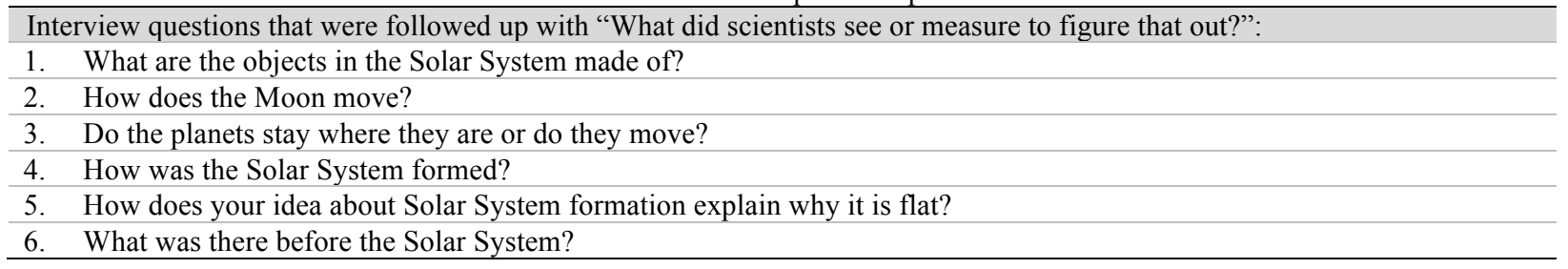

Five members of the research team individually coded two different videos each, selected as a representative sample across grade levels. Following this, we refined and finalized the codes by studying and discussing these 10 videos ( $24 \%$ of data set) as a group. The codes had to be general enough that they could be applied to the different contexts in which the questions were asked (e.g., about planetary motion or Solar System age). Codes with similar themes were then grouped together into categories. We used the team's analysis of this group of interviews to train a single member of the team, part of the original team who developed the codes and categories, to apply those codes to the remaining interviews. Once this system of categories and codes had been applied to the interviews, we looked for broad patterns about how astronomers gather evidence about the nature of the objects in the Solar System.

\section{RESULTS}

In this section, we describe the broad categories we used to code student ideas about how astronomers learn about the Solar System, describe the general pattern we saw across the entire sample of student responses, and share some individual examples that illustrate our findings. The categories used to organize student ideas and the frequency of students with a response in that category are presented in Table 2. A student was included in a category if they were coded as having used a particular response at least one time among the several interview questions listed in Table 1. 
Table 2. Top-level categories and frequency of students coded in that category for student ideas about science practices in astronomy

\begin{tabular}{l|c}
\multicolumn{1}{c}{ Category (with abbreviations) } & $\begin{array}{c}\text { Frequency coded in the category } \\
(\mathbf{N}=\mathbf{4 2})\end{array}$ \\
$\begin{array}{l}\text { Scientists find out about }<\text { blank }>\text { by making or using observations } \\
\text { (Using Observations) }\end{array}$ & 34 \\
\hline $\begin{array}{l}\text { Scientists find out about }<\text { blank }>\text { by space exploration } \\
\text { (Space Exploration) }\end{array}$ & 29 \\
\hline $\begin{array}{l}\text { Scientists find out about }<\text { blank }>\text { through investigations, experiments or some kind of test } \\
\text { Investigations) }\end{array}$ & $(69 \%)$ \\
\hline $\begin{array}{l}\text { Scientists find out about }<\text { blank }>\text { by making inferences from observations } \\
\text { Inferences) }\end{array}$ & $(45 \%)$ \\
\hline $\begin{array}{l}\text { Scientists find out about }<\text { blank }>\text { by inferring from a casual model } \\
\text { (Casual Model) }\end{array}$ & 13 \\
\hline $\begin{array}{l}\text { There is no way for scientists to find out about }<\text { blank }> \\
\text { (No Way) }\end{array}$ & $(31 \%)$ \\
\hline
\end{tabular}

Within each of these categories was a range of codes that captured student ideas at a finer grain-size. The most common responses were found in categories Using Observations and Space Exploration, so we will describe those responses in the most detail. The other categories also included some relevant ideas to understanding students' thinking about astronomical practices, so they are described in more detail in separate sub-sections.

\section{Using Observations}

The majority of the students (81\%) gave an answer that was coded as Using Observations in one or more of the interview questions. Student ideas in the category of astronomers making or using observations were subdivided further using codes for naked eye observations, general telescope use, ground-based telescope use, space-based telescope use, astronomers taking multiple observations over time, or taking photographs for later analysis. While these are all practices used by astronomers, many students only provided enough detail to imply that astronomers make observations. Since these codes were generated from the full set of student responses, they did influence our perspective of what practices we considered to be the practices of astronomers and planetary scientists. No student in our sample included a response that suggested you could observe or investigate the properties of a lunar or Martian meteorite, for example, in order to study those objects. Thus, we did not develop codes in this category or in the Investigations category for this type of response.

Students often described scientifically correct ideas about how the planets orbit the Sun and how the Moon orbits the Earth; however, when they were asked how scientists know these objects move this way, several implied that simply watching the Moon with the naked eye reveals its orbital motion. Although naked eye observations are enough to reveal the Moon orbits the Earth, it requires sophisticated spatial reasoning to be able to describe why the Moon's changing appearance is a result of its orbital motion (Plummer, 2014). Over the course of a month, the Moon will rise and set at different times while simultaneously changing its phase. The Moon's changing location with respect to the Earth and the Sun is responsible for these observable changes. Only one student suggested the Moon's orbital motion could be inferred from this changing appearance. However, that type of answer where a student both mentions naked eye observations of the Moon and how this changing appearance can be used to infer its motion would be coded as either Using Observations + Inferences or Using Observations + Causal Model depending on the detail they provided about how they inferred its motion; as shown in Table 2, far fewer of the students gave answers in those additional categories.

There were students who demonstrated more sophisticated understanding of how astronomers use observations to study planetary motion. Almost one-third of the group (12 students) provided answers that suggested that taking photographs of the planets at several times could reveal their motion, which is still the method used by research astronomers today to discover new Solar System objects. 
This dramatic difference between the number of students being coded in the Using Observations category by itself compared to that category+Inferences or Causal Model suggests to us that the students interviewed did not understand how systematic study of patterns in astronomical observations can be used to infer complex scientific models, such as the motion of the Moon and planets.

When the interviewer asked about planetary composition, students employed naive reasoning about how observations reveal this planetary property. For example, several students said you can learn about a planet's composition by looking through a telescope or by taking a photograph. One student referred to having seen pictures that led them to conclude that Uranus is covered with "blue ash", while Mars is covered with "red ash" in their answer to this prompt. However, a typical image of a planet reveals little about its composition. Much of astronomers' understanding of planetary composition comes from analyzing the reflected light from an object in images taken through multiple color filters or from collecting a spectrum of a planet from an Earth-based telescope or a robotic orbiter. Only two collegelevel students mentioned spectroscopy as a method to determine a planet's composition, but struggled to explain in detail how a spectrum can reveal an object's composition. While it is common for college students in introductory astronomy courses to have an experience with spectroscopy in their astronomy class (Slater et al., 2001), this did not help these particular students reach a more sophisticated level of explanation for how astronomers determine planetary composition. It is also unlikely that middle and high school students would have had a similar experience with spectroscopy and this may explain why none of them were including spectroscopy in their responses.

The results for the category Using Observations indicate that few students have much familiarity with the types of observations made by astronomers as they plan and carry out their investigations (practice 3 from the Framework), but they made frequent mention of making observations as a general scientific practice. Thus, it may be productive for teachers to build on this aspect of students' thinking towards a more normative understanding of how astronomers make observations.

\section{Space Exploration}

More than two-thirds (69\%) of students interviewed gave a response coded in Space Exploration. Student ideas about how scientists use space exploration to investigate the Solar System included descriptions of both robotic and humancrewed spaceflight, and in both cases they discussed missions with and without sample return to Earth. These responses seemed to be influenced by media coverage of the many recent lander and rover missions to Mars as many students directly mentioned rovers. For context, Mars Curiosity landed on Mars in August 2012, and these interviews were conducted during the spring and summer of 2012. Twenty-one of the students (50\%) suggested that learning planetary composition requires either a robotic mission or a human spaceflight mission to a planet without indicating this was possible to do using telescopes. Only $11(26 \%)$ of the students suggested this could be done with telescopes, and three of those responses were from the college students interviewed, who had received direct instruction about this concept. Twelve students (29\%) indicated these missions required returning physical samples of the planet to Earth, and $8(19 \%)$ of those suggested astronauts were required to obtain the samples. The NASA Apollo missions successfully returned samples of lunar material, two less well-known missions (Stardust and Hayabusa) returned microscopic samples from a comet and an asteroid, and the NASA Genesis mission returned particles from the Solar wind. No planetary material has ever been obtained directly from a planet and returned to Earth, although there are meteorites that have been discovered serendipitously on Earth that we believe have come from Mars. Furthermore, astronauts have never visited any Solar System object besides the Moon. Planetary scientists make extensive use of meteoritic studies in their work, including the study of meteorites believed to be made of lunar or Martian material. No student in our sample made reference to samples found on Earth, though; in every case where a student discussed studying a sample they indicated that it had to be physically retrieved by a robot or an astronaut. We were surprised to find, however, that eight students (19\%) believe that humans have visited a variety of Solar System bodies beyond the Moon. Although this is a relatively small percentage of the whole sample, the prevalence of this particular naive idea about Solar System exploration was revealing, because it seems to indicate that students do not appreciate how much of astronomical practice requires study of objects at a large distance.

The prevalence of these non-normative ideas about human spaceflight and sample return seems to indicate that students are not being provided with good instructional opportunities to learn how astronomers can infer the 
composition of an object by analyzing its light. Instead, students seem to rely on prior experiences in school, such as using experiences investigating the composition of a material like a mineral specimen, for example, when asked how astronomers learn the composition of Mercury. Similarly, when asked how scientists know the age of the Solar System, one student described visiting a planet and doing an experiment similar to those that are done to date fossils. Although this student described an experimental test that could be performed to determine the age of the Solar System, he still believed it required an astronaut land on a planet. Among the eight students who made claims about human sample return in their interviews, seven of them were middle-school students. However, one college student also gave this answer, suggesting this conception that astronauts conduct sample return missions is not entirely a function of the age or prior instruction of the student.

Category Space Exploration led us to the same conclusion as in category Using Observations; although students appealed to rovers and landers, which are used by astronomers in carrying out investigations, they believe that human spaceflight and sample return are far more prevalent than they actually are in the field.

\section{Investigations}

Student responses were most often coded in the Using Observations and Space Explorations categories by themselves and less frequently were coded as both Observations and Investigations or Space Explorations and Investigations but rarely Investigations by itself. With this system of categories, we anticipated this particular association; if a student describes an investigation ( $45 \%$ did), that investigation had to rely on some type of observation. The same type of reasoning applies for the Inferences (31\%) and Causal Models (12\%) categories described in the next two sections. For this reason, we consider these last three categories to be more sophisticated ideas than those where a student just described an observation without also describing how that observation could, for example, lead to an investigation with some evidence-based result. The variety of responses in these three categories was not as rich as in the observations and space exploration categories, and because students were really stretching their understanding, their ideas were more often partially correct and did not approach what our team considered to be expert understanding. Given that the college students were selected in order to provide more sophisticated answers, we expected them to be coded in these categories more frequently, and they were. However, 14 of the 19 responses coded in the investigations category were from middle-school and high-school students.

In the responses coded as Investigations, the ideas students expressed were often similar to those in Observations and Space Exploration with only slightly more elaboration that suggested more sophisticated thinking. For a student response to have been identified as an investigation, they had to provide some detail about how their observation would provide insight about a planet. For example, one student described a crewed spaceflight mission to a planet, but then added that scientists could, once there, do an experiment like those done on Earth to analyze dinosaur bones. So this additional level of detail about the experiment led us to categorize this as an investigation, rather than just an observation that somehow provides an answer. Another idea that a student proposed in this category was to create a tether between the Earth and the Moon and to follow the behavior of the tether to determine if the Moon was moving or stationary. While this is implausible because of the difficulty of such an experiment, it was a valid procedure that could in theory be performed. This tether idea is another example of a student proposing a method that is very different from the typical astronomy practice of analyzing observations made at a distance with a telescope.

\section{Inferences}

We coded as Inferences those examples where students provided details about how a particular observation would provide evidence one could use to infer something about a Solar System object. Among the 13 responses included in this category, four were from middle-school students, five from high-school students, and four were given by college students. For example, one partially correct and productive idea was coded as Inferences and was expressed by two students; they said that if a planet appears blue in a photo (like Neptune), that this implies it must have water. Students familiar with images of Earth from space know that the blue color of Earth is caused by water, and they made this connection to Neptune, even though it is incorrect. This type of inference is indicative of the type of argument that astronomers make from the evidence contained in an astronomical image, even though Neptune is blue for a different reason. This is an example of a student showing some understanding of how astronomers analyze and interpret data 
on astronomical objects, and this could be leveraged by a knowledgeable instructor to move a student to more expert understanding of this practice.

\section{Causal models}

We coded as Causal Models those student ideas where they explicitly identified a causal model commonly used in astronomy as part of their scientific reasoning. For example, when asked how astronomers know the Moon is in motion, one middle-school student discussed a thought experiment that invoked a sophisticated understanding of our model for how gravity works to explain how we know the Moon must move. This was coded as Causal Models, because it required describing such a model to explain the Moon's motion. This type of reasoning was rare, though, with the only exception being from the college students, most of whom referred to various scientific models in their responses. Besides the one middle-school student described above, none of the other middle- or high-school students in the sample invoked this type of reasoning. Given that developing and using models is one of the other scientific practices highlighted in the Framework, these interviews suggest that students are rarely learning this skill in current astronomy instruction.

\section{CONCLUSIONS}

The interviews that we conducted had the dual goal of informing a learning progression for student understanding of Solar System astronomy (Plummer et al., 2015) and determining student ideas about how astronomers engage in scientific practices to study the Solar System. We found that many students had ideas about how astronomers might observe or explore using crewed or robotic missions to the worlds in the Solar System. However, many of their ideas did not include insight into how astronomers reach-evidence based conclusions using those observations. Instead, they often described an observation and said that the result of that observation was knowledge about the Solar System without providing further detail about how that particular dataset or measurement would be used to create that knowledge. A smaller number of students described observations or space exploration and coupled those descriptions with more sophisticated ideas about how astronomers can interpret the data generated from those observations into insight about the Solar System. However, these more sophisticated ideas were often only partially correct and were not examples of expert understanding of the practices of astronomy. It is interesting to note that when students described investigations that might be carried out, these often were modeled after the types of investigations they might have done or heard about when studying geoscience or physics, such as the dinosaur bone analogy described in sub-section 3.3.

The conclusion we draw from these findings is that typical astronomy instruction does not engage students in the practices astronomers use when planning and carrying out astronomical investigations or interpreting astronomical data obtained from these investigations. Thus, when students are asked about the practices of astronomy, they draw from other cultural experiences or classroom experiences in other science domains to formulate their responses.

When we analyzed the student responses relevant to the practice of planning and carrying out investigations as enumerated in the Framework, students seemed to have some familiarity with how astronomers plan and carry out one type of astronomical investigations: space exploration. The idea of sending a human or a robot to obtain a physical sample from a planet is a valid method used and has been accomplished for a few selected objects. However, the interviews revealed that students showed very little understanding of how astronomers plan and carry out investigations with telescopes.

A few student responses indicated that they were somewhat familiar with methods scientists might use to analyze and interpret data. For example, the color of a planet seen in a photo can indicate its composition and astronomers determine one estimate for the age of the Solar System using the same techniques used to determine the age of fossils, as several students described in interviews. Our interview protocol gave students the opportunity to appeal to other models of astronomical phenomena in their responses, but very few employed this type of reasoning.

These results have implications for instruction that supports the goals of the Next Generation Science Standards. Students need instruction that is designed to reveal the limits of human spaceflight and sample return missions while 
simultaneously emphasizing how much more astronomers rely on telescopic observations from a large distance to study the Solar System. Sadler (1992) noted in his dissertation that prior to instruction, many high-school students believe the stars to be found inside the boundaries of the Solar System. We saw this alternative conception appear among students in this study, too. Thus, it should not be surprising that students who do not understand the scale of the Solar System and the stars assume that we can study these objects using technology, like rovers, which has been used so successfully to study Mars. This finding suggests that engaging students in instruction designed to have them consider the set of objects that can be studied with a given technique, e.g., robotic orbiters, robotic landers, or telescopes, would be an important addition to an astronomy curriculum. This type of investigation requires students to understand the scale of the Solar System and the nearby stars, which is a common topic in many K-12 astronomy units and is important to understanding the "Earth's place in the Universe" disciplinary core idea in the NGSS.

Further, our findings imply that students would likely benefit from more direct instruction in the use of telescopes to obtain their own astronomical images of distant objects. Students may have appealed to physical sample return in their interview responses because they had little or no experience with obtaining observations of objects at a distance with a telescope equipped with an instrument for capturing data. The primary barrier to engaging students in optical telescope use is that instruction always occurs during the day and astronomical observations with these types of telescopes require them to be used after dark, which is not the case with most of the tools used in other scientific domains. Even when students do have the opportunity to do their own astronomical observing with a telescope, this is frequently eyepiece observing that does not result in the acquisition of a digital image or spectrum of the object being observed. Thus, most students have limited opportunities to directly experience the techniques used by astronomers to collect data and gather evidence. Slater and colleagues (2014) proposed a hypothetical learning progression that can be used to design new curriculum for astronomy classes that incorporate remote or robotic telescopes to overcome this barrier to access to telescopes during the typical school day. This learning progression may be useful as a framework for studying student learning in classes with access to telescopes to reveal if direct use does indeed influence their ideas about the practices of astronomy.

A promising future direction for research of this type would be to study the results of curriculum interventions that engage students in the use of robotic telescopes and how these may influence the fusion of both astronomy content and practice. In particular, we see already how students could benefit significantly from an astronomy curriculum which includes an investigation of the types of methods astronomers use to study nearby planets (e.g., Mars), distant planets (e.g., Neptune), and stars, which synthesizes the ideas of scale of the Universe with the practices of astronomy. While this type of study may reveal how students are contextualizing science practices from the NRC Framework into the specific field of astronomy, we know that students will be studying and engaging in these practices in very different ways in different sciences across their entire K-12 curriculum. Thus, with additional, similar studies on how students are experiencing and learning about these practices in other grades and other sciences, we may be able to understand more generally how they synthesize their experiences with practices in different disciplines into a coherent understanding of science that crosses disciplines.

\section{ACKNOWLEDGEMENTS}

This work was funded by the National Science Foundation, award \#DUE-0962792. We thank the anonymous reviewers for their helpful suggestions to improve the quality of this work.

\section{AUTHOR BIOGRAPHIES:}

Christopher Palma is a senior lecturer and the head of undergraduate programs for the Department of Astronomy \& Astrophysics at the Pennsylvania State University. He is part of the leadership team and the astronomy learning progression research team for the Earth and Space Science Partnership at Penn State. E-mail: cpalma@psu.edu (contact author).

Julia Plummer is Associate Professor of Science Education in the Department of Curriculum \& Instruction at Penn State University. Prior to this role, she spent more than a decade teaching children and adults in planetariums. Her research interests include learning progressions, spatial thinking, and science practices in astronomy, primarily at the 
preK-12 level. She conducts research across settings, including both informal spaces and classroom environments. E-mail: jdp17@psu.edu

KeriAnn Rubin is a middle school STEM teacher at St. Ann School in Stoughton, Wisconsin. Prior to this role, she completed her Master's Degree at Pennsylvania State University in Curriculum \& Instruction: Science Education after teaching for two years at St. Patrick School in Newry, Pennsylvania. She is a member of The National Science Teacher Association and the Wisconsin Society of Science Teachers. E-mail: keriannrubin@gmail.com

Alice Flarend is a Nationally Board Certified Physics teacher with over twenty years of teaching experience and is completing a doctorate in Curriculum and Instruction at Penn State University with a research interest in teacher professional development. She is a member of the Physics Teacher Resource Agent Program of the American Association of Physics Teachers, offering physics teacher professional development. Prior to becoming a teacher, Alice studied nuclear engineering with an emphasis on radioanalytical techniques. E-mail: ama177@psu.edu

Yann Shiou Ong is a Ph.D. candidate at PSU, Department of Curriculum and Instruction (Science Education). Her research interests include scientific inquiry, argumentation, epistemic cognition, and collaborative learning. She received a M.Sc. in Science Education from the University of Bristol, UK. She was formerly a middle and high school physics teacher in Singapore. E-mail: yuo103@psu.edu

Scott McDonald is an Associate Professor of Science Education at The Pennsylvania State University and Director of the Krause Innovation Studio in the PSU College of Education. He received his undergraduate degree in Physics with a focus on Astronomy and Astrophysics. He was a high school Physics teacher for six years before returning for a Ph.D. in Learning Technologies and Science Education at the University of Michigan. E-mail: smcdonald@psu.edu

Chrysta Ghent is pursuing her Ph.D. in Curriculum and Instruction at Penn State University with an interest in planetarium educator professional development. Prior to attending PSU, she was a certified middle and high school Earth and Space Science teacher and taught high school Biology in Maryland. E-mail: cag1030@psu.edu

Tim Gleason is a student at Penn State University. He is enrolled in a concurrent Master's of Education program specializing in secondary science education as well as an undergraduate program in Planetary Science \& Astronomy. He will soon have his Pennsylvania teaching certification in Earth and Space Science and hopes to teach that subject at the high school level. E-mail: tgg119@psu.edu

Tanya Furman is Professor of Geosciences and Associate Vice President and Associate Dean for Undergraduate Education at PSU. Her research focuses on the evolving internal structure of the Earth using geochemical evidence from lavas erupted in areas of continental rifting. She is also part of the research team developing learning progressions for student understanding of plate tectonics. She leads the Earth and Space Science Partnership, under whose auspices this work was performed. E-mail: tfl3@psu.edu

\section{REFERENCES}

Brickhouse, N. W., Dagher, Z. R., Shipman, H. L., \& Letts, W. J. (2002). Evidence and warrants for belief in a college astronomy course. Science \& Education, 11(6), 573-588.

Camacho, A., Holt, R., Marley, S., \& Roberts, S. (2009). Robotic telescopes-Classroom activities at the cutting edge of science. Space Science Reviews International Journal, 90, 73-82.

Deeb, E., Rebull, L. M., Black, D. V., Gibbs, J., \& Larsen, E. (2015, January). NITARP: Measuring the effectiveness of an authentic research experience in secondary astronomy education through concept mapping. In American Astronomical Society Meeting Abstracts (Vol. 225).

Eberbach, C., \& Crowley, K. (2009). From everyday to scientific observation: How children learn to observe the biologist's world. Review of Educational Research, 79(1), 39-68.

Ferlet, R., \& Pennypacker, C. R. (2006). The Hands-On Universe Project. In Organizations and Strategies in Astronomy Volume 6 (pp. 275-286). Springer Netherlands.

Fields, D. A. (2009). What do students gain from a week at science camp? Youth perceptions and the design of an immersive, research-oriented astronomy camp. International Journal of Science Education, 31(2), 151-171. 
Gehret, L., Winters, W., \& Coberly, S. (2004). The internet telescope: Remote observing for the city astronomy class. Astronomy Education Review, 3(2), 170-177.

Gershun, D.C., Slater, T.F., \& Berryhill, K.J. (2014). Mixed-methods study that examines nine science teachers' perceptions of Slooh robotic telescope for teaching astronomy. Latin American Journal of Astronomy Education RELEA, 17, 7-37.

Gould, R., Dussault, M., \& Sadler, P. (2006). What's educational about online telescopes?: Evaluating 10 years of MicroObservatory. Astronomy Education Review, 5(2).

Hoban, S., Farrell, N., Rathod, P., Sachs, J., Sansare, S., Yesha, Y., ... \& Mayo, L. (2006). Virtual telescopes in education. Journal of Digital Information, 2(4).

Kesidou, S., \& Roseman, J. E. (2002). How well do middle school science programs measure up? Findings from Project 2061's curriculum review. Journal of Research in Science Teaching, 39(6), 522-549.

Lowman, P. (2002). Exploration Science: A case history from Earth orbit. The Science Teacher, 69(7), 24-30.

Marschall, L. A., Snyder, G. A., \& Cooper, P. R. (2000). A desktop universe for the introductory astronomy laboratory. The Physics Teacher 38(9), 536-537.

Miller, B. W., \& Brewer, W. F. (2010). Misconceptions of astronomical distances. International Journal of Science Education, $32(12), 1549-1560$.

NGSS Lead States. (2013). Next generation science standards: For states, by states. National Academies Press. Retrieved from http://www.nextgenscience.org/

National Research Council (2007). Taking science to school: Learning and teaching science in grades K-8. Washington, D.C.: National Academies Press.

National Research Council (2012). A Framework for K-12 Science Education: Practices, Crosscutting Concepts, and Core Ideas. Washington, D.C.: National Academies Press.

Palen, S., \& Proctor, A. (2006). Astronomy in the K-8 core curriculum: A survey of state requirements nationwide. Astronomy Education Review, 5(1), 23-35.

Plummer, J. D. (2014). Spatial thinking as the dimension of progress in an astronomy learning progression. Studies in Science Education, 50(1), 1-45.

Plummer, J. D., Palma, C., Flarend, A., Rubin, K., Ong, Y. S., Botzer, B., ... \& Furman, T. (2015). Development of a learning progression for the formation of the solar system. International Journal of Science Education, 37(9), 1381-1401.

Reiff, R., Harwood, W. S., \& Phillipson, T. (2002). A Scientific Method Based upon Research Scientists' Conceptions of Scientific Inquiry.

Sadler, P. M. (1992). The initial knowledge state of high school astronomy students (Doctoral dissertation, Harvard University). Retrieved from http://www.cfa.harvard.edu.

Sadler, P. M., Gould, R. R., Leiker, P. S., Antonucci, P. R. A., Kimberk, R., Deutsch, F. S., ... French, L. (2001). MicroObservatory Net: A network of automated remote telescopes dedicated to educational use. Journal of Science Education and Technology, 10(1), 39-55.

Slater, T., Adams, J. P., Brissenden, G., \& Duncan, D. (2001). What topics are taught in introductory astronomy courses?. The Physics Teacher, 39(1), 52-55.

Slater, T. F., Burrows, A. C., French, D. A., Sanchez, R. A., \& Tatge, C. B. (2014). A proposed astronomy learning progression for remote telescope observation. Journal of College Teaching \& Learning, 11(4), 197-206.

Songer, N. B., \& Gotwals, A. W. (2012). Guiding explanation construction by children at the entry points of learning progressions. Journal of Research in Science Teaching, 49(2), 141-165. 